\title{
Single Metal of Silver Nanoparticles in the Microemulsion for Recyclable Catalysis of 4-Nitrophenol Reduction
}

\author{
Shizhen Zhao, Liping Duan, Chunlin Xiao, Li Li, Fang Liao* \\ Chemical Synthesis and Pollution Control, Key Laboratory of Sichuan Province, School of Chemistry and Chemical \\ Industry, China West Normal University, Nanchong, 637002, China. \\ Email: liaozhang2003@163.com
}

\begin{abstract}
Size control of silver nanoparticles (Ag NPs) to improve monodispersity and recyclability is crucial for application in nanocatalysts. Hence, a novel and effective protocol for in-situ synthesis of $\mathrm{Ag}$ NPs in the microemulsion was proposed. The surfactant-stabilized microcavity in microemulsion can provide a nanoscale reactor that limits nucleation, growth, and agglomeration of the particles. Ag NPs of 540-640 nm were successfully grown and exhibited excellent catalytic activity with the apparent rate constant (k) of $0.59 \mathrm{~min}^{-1}$ and the activation energy (Ea) of $23.03 \mathrm{~kJ} \mathrm{~mol}^{-1}$ toward the reduction of 4-nitrophenol. Moreover, the catalyst could be easily recycled and showed excellent reusability after 6 cycles. So, the silver nanoparticles can be extended to an important metalcatalyzed reduction in chemical industry, which is of great significance for the sustainable development.
\end{abstract}

Keywords: Silver nanoparticles, microemulsion, 4-NP, catalytic

\section{Introduction}

As one of the most promising functional materials, silver nanoparticles (Ag NPs) in recent years are appreciated for their unique and excellent optical, antimicrobial, electrochemical, and also catalytic properties [1-3]. Thus, Ag NPs have catalytic activity for many organic reactions, such as the reduction reaction of nitro compounds [4], ethylene epoxidation [5] and organic dyes [6]. Compared with other metal nanoparticles with catalytic activity, such as Au, Pd and Pt nanoparticles [7-9], Ag NPs can be prepared more readily and inexpensively [10]. However, it usually suffers from irreversible aggregation when Ag NPs were synthesized [11]. Ag NPs normally incline to grow and form big agglomerates owing to their excellent surface energy, thus resulting in reduction of their surface area $[12,13]$.

Recently, various synthesis methods have been explored to prepare Ag nanomaterial with different kinds of structures and applications such as hydrothermal treatment [14-19], microemulsion and reverse micelles $[20,21]$ chemical vapor deposition, electrodeposition and the microwave method [22]. Especially, microemulsion synthesis has been demonstrated to be a very promising method due to its precise control on morphology and dimension of nanoparticles [22]. From the viewpoints of colloid and interface chemistry, there are two types of microemulsions, reversed (water/oil) and direct (oil/water) microemulsion [23-25]. These systems are transparent, isotropic liquid media composed of discrete, thermodynamically stable [20]. The surfactant-stabilized microcavity in microemulsion can provide a nanoscale reactor that limits nucleation, growth, and agglomeration of the particles [22]. Accordingly, we could use this method to get $\mathrm{Ag}$ NPs with a controlled dimension.

As we all know that 4-nitrophenol (4-NP) shows one of the most notorious toxic pollutants in the industrial effluents [26]. 4-aminophenol (4-AP) is very useful and important in many applications including analgesic and antipyretic drugs, photographic development, corrosion inhibition, anticorrosion lubrication, hair-dyeing agent etc [27]. Therefore enormous current interests have arisen in the development of catalysts for reduction of the 4-NP to 4-AP [28-30]. Traditional methods for this process usually involve reduction/catalytic hydrogenation [31-35]. Considering these aspects, sizecontrolled synthesis of Ag NPs for 4-NP transformations in a simple, cost-effective, and selective manner is highly demanding [26].

We herein report our new efforts to synthesize Ag nanoparticles by the microemulsion method. Through our research, most of catalysts were alloy or compound of silver nanoparticles traditionally. A single metal of Ag NPs were manufactured in our work through a simple and fast method. In this system, o-phenylenediamine (oPD) was used as a weak reductive agent, who has been employed to produce silver 
nanostructure in previous reports [17]. In this study, the Ag NPs were high-yield synthesized by mixing oPD and microemulsion which was composed of n-hexane, ethyl alcohol and distilled water. And different proportion of this composes was discussed in our work. The resulting Ag NPs showed excellent catalytic activity for 4-NP to 4-AP. The highly efficient and stable catalytic performance under sunlight irradiation makes them promising alternatives to the conventional UV light or visible-light energized photocatalysts.

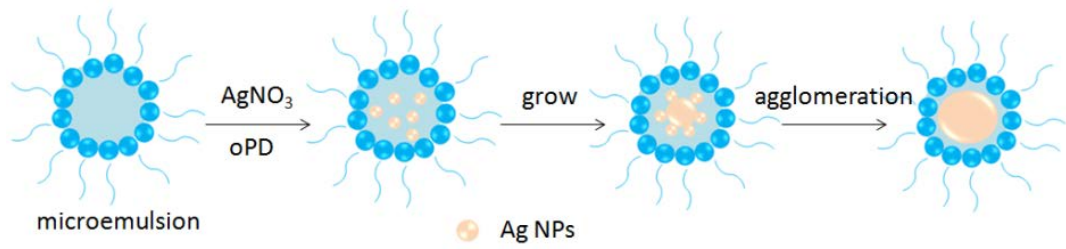

Scheme 1. Schematic illustration of in situ generation of Ag NPs in microemulsion.

\section{$2 \quad$ Materials and Methods}

\subsection{Materials}

$\mathrm{AgNO}_{3}$ (99.8\%), o-phenylenediamine (oPD, 99.0\%), n-hexane (97\%), ethyl alcohol (EtOH, 99.7\%), 4-Nitrophenol (4-NP, 99.5\%), $\mathrm{NaBH}_{4}(98 \%)$ were purchased from kelong (chengdu, China) and used as received without further purification. The ultrapure water was purified using a Millipore $(18.2 \mathrm{M} \Omega \cdot \mathrm{cm})$ system and used in all experiments.

\subsection{Synthesis of Ag NPs}

Typically, according to a certain proportion of microemulsion, the volume ratio of N-hexane, ethanol and water was 10:1:2, 10:10:2, 10:5:2 respectively. Then $\mathrm{AgNO}_{3}$ and oPD was added to the above solution with the molar ratio 1:1. The mixed solution was stirred for $2 \mathrm{~h}$ at room temperature, and the color of the solution gradually changed from light yellow to brown. Products were collected by centrifugation, and sequentially washed with EtOH and water to remove adsorbed organics. The silver precipitates were washed with water by centrifugation twice, and the resulting precipitates were dried at $60^{\circ} \mathrm{C}$ for $12 \mathrm{~h}$ in vacuum for further use.

\subsection{Characterization Methods}

Scanning electron microscopy (SEM) measurements were made on a JSM-6510 (Rigaku, Japan) operated at an accelerating voltage of $20 \mathrm{kV}$. UV-vis spectra were obtained on a UV-7502PC Spectropheotometer. The crystal structure and purity of Ag NPs was characterized by powder X-ray diffraction (XRD) patterns recorded on a Dmax/Ultima IV (Japan Rigaku).

\subsection{Catalytic Reduction of 4-Nitrophenol}

For evaluating the activity of our Ag NPs as an efficient catalyst, the reduction of 4-NP by NaBH4 was carried out in experiments as follows. Since the catalytic degradation of 4-NP follows pseudo-first-order kinetics, the experiment was done taking $20 \mathrm{~mL}$ of $2 \mathrm{mM} 4-\mathrm{NP}$ solution in $20 \mathrm{~mL}$ of $0.25 \mathrm{M} \mathrm{NaBH}_{4}$ solution, leading to an immediate color change from light yellow to yellow-green. $0.02 \mathrm{~g}$ of Ag NPs was then added to the mixture as catalyst. The reaction was carried out at $25^{\circ} \mathrm{C}$ with continuous stirring to ensure homogeneous mixing of the reactants and distribution of the catalyst. And then $600 \mu \mathrm{L}$ mixed solution was taken in a standard quartz cuvette of $1 \mathrm{~cm}$ path length with $3 \mathrm{~mL}$ distilled water. The UV-vis absorption spectra were then recorded at RT in a scanning range of 250-500 nm. For studying the effect of catalyst dose, the amount of Ag NPs catalyst was varied $0.01 \mathrm{~g}-0.03 \mathrm{~g}$; however, volume and concentration of $4-\mathrm{NP}$ and $\mathrm{NaBH}_{4}$ were kept constant (20 mL of $2 \mathrm{mM}$ and $20 \mathrm{~mL}$ of $0.25 \mathrm{M}$, respectively). In a similar way, the temperature was varied $0-95^{\circ} \mathrm{C}$. 


\section{$3 \quad$ Results and Discussion}

\subsection{Characterization of Ag NPs}

The fabrication of Ag NPs is illustrated in Scheme 1. In the first stage of silver nanoparticles, the surface area of Ag NPs is large, so it will spontaneously aggregate together. However, under the condition of microemulsion, Ag NPs grow, and agglomerate in a certain size and shape.

Fig. 1a-c represents the typical low magnified SEM images of Ag NPs which the volume ratio of N-hexane, ethanol and water was 10:1:2, 10:10:2, 10:5:2 respectively. As we could see, Ag NPs suffered from serious aggregation with a small amount of ethanol (Fig.1a). In the microemulsion, ethanol plays a role of surfactants. So in the case of less ethanol, a serious aggregation was appeared. As the increase of ethanol, Ag NPs emerged granulum structure without any aggregation. However, the particle size of Ag NPs was not uniform when the volume ratio was 10:10:2 (Fig. 1b). This was probably because of forming uneven cavity in the nanoscale reactor of microemulsion. Fig.1c shows the low magnification SEM image of Ag NPs the volume ratio of which was 10:5:2 and the high magnification SEM image were shown in Fig. 1d. Obviously, most of the products were granulum structure with a size distribution of 580-600 nm. The inset showed particle size distribution of Ag NPs, demonstrating the diameter of Ag NPs was 540-640 nm. Therefore, we thought that the microcavity in microemulsion could provide a homogeneous nanoscale reactor with the volume ratio 10:5:2. The composition of the Ag NPs was confirmed by EDS spectrum in Fig.1e. The inset showed the concentration corresponding to $\mathrm{Ag}$ and $\mathrm{O}$ elements. The XRD pattern of $\mathrm{Ag}$ NPs was shown in Fig.1f. The spectra of sliver nanocrystals displayed specific peak at $2 \theta=38.3^{\circ}, 44.5^{\circ}$, $64.6^{\circ}$ and $77.5^{\circ}$, corresponding to the (111), (200), (220) and (311) crystallographic planes, respectively.

\subsection{Catalytic Reduction of 4-Nitrophenol}

The reduction of 4-nitrophenol to 4-aminophenol by $\mathrm{NaBH}_{4}$ reducing agent was chosen to evaluate the catalytic performance of the Ag NPs. The progression of the reduction reaction was monitored by UV-vis absorption spectroscopy. An aqueous solution of 4-NP is light yellow in color, which shows absorption at $317 \mathrm{~nm}$. The peak redshifted from ca.317 to $400 \mathrm{~nm}$ after the addition of $\mathrm{NaBH}_{4}$ and the color changed from light yellow to deep yellow. This color change can be attributed to an increase in the alkalinity of the solution, which results in the formation of the 4-nitrophenolate ion $\left(\mathrm{C}_{6} \mathrm{H}_{4} \mathrm{NO}_{3}^{-}\right)$[36].

Fig. 2a-c shows the time-dependent UV-vis absorption spectra with the Ag NPs catalyst added. With the volume ratio of 10:1:2, 10:10:2, 10:5:2 in microemulsion, the reduction reaction was $12 \mathrm{~min}, 9 \mathrm{~min}$ and 7 min respectively. Due to suffer from a serious aggregation, Ag NPs had a low efficiency when the volume ratio was 10:1:2 (Fig. 2a). However, owing to nonuniform of particle size, the reaction was not complete after 7 min (Fig. 2b). As a representative case of the catalyst, this reduction reaction was completed in 7 min (Fig. 2c), as indicated by a fast color change of the reaction solution from yellow to colorless. From the absorption spectra, it was noted that the intensity of the absorption peak at $400 \mathrm{~nm}$ dramatically decreases and, at the same time, a new peak at $298 \mathrm{~nm}$ started to rise due to the conversion of 4-NP to 4-AP.

Fig. 2d shows $\ln \left(A_{t} / A_{0}\right)$ versus reaction time for the reaction of 4-NP with Ag NPs as catalysts, where $A_{0}$ and $\mathrm{A}_{t}$ are the initial absorption intensity and absorption intensity of 4 -NP at time $t$, respectively. It was obvious that the good linear relationships of $\ln \left(\mathrm{A}_{t} / \mathrm{A}_{0}\right)$ versus reaction time were obtained, suggesting that the reactions followed a pseudo-first-order kinetics reaction. The pseudo-first-order rate constant of the reaction catalyzed by the $\mathrm{Ag}$ NPs catalyst was calculated by measuring the disappearance of the characteristic peak, viz., the concentration of 4-NP quantitatively at $400 \mathrm{~nm}$ collected at given intervals. The rate constant can be calculated using the formula

$$
\ln \left(\mathrm{A}_{\mathrm{t}} / \mathrm{A}_{0}\right)=-\mathrm{k}_{\text {app }} \mathrm{t}
$$

where $k_{\text {app }}$ is the apparent rate constant, $t$ is the reaction time, $A_{0}$ is the initial concentration of 4-NP, and $A_{t}$ is the concentration of 4-NP at time $t$ [30]. As expected, a linear relationship between $\ln \left(A_{t} / A_{0}\right)$ and reaction time was obtained. Thus, the rate constant was estimated from the slop of the best-fit line as ca.0.59 $\mathrm{min}^{-1}$, which was much higher than that of other catalysts reported elsewhere (Table 1) [1, 30, 37-42]. Due to the fact that our catalyst was of low cost, easy to synthesize, and highly efficient, it was beneficial over those from other reports. 
The effects of Ag NPs catalyst dosing were investigated by varying the concentration of catalyst, while keeping other parameters, such as the concentration of 4-NP and $\mathrm{NaBH}_{4}$, constant (Fig. 3a). The reaction rate generally increased with the mass of catalyst. This observation is consistent with the view that an increase in the total surface area and number of reaction sites enhances the catalytic activity [43]. However, when the mass of catalyst was more than $0.02 \mathrm{~g}$, the average rate of increase was decreased obviously (Fig. $3 \mathrm{~b})$.

In order to investigate $\mathrm{Ag}$ NPs catalyst for 4-NP reduction reaction, the optimal reaction temperature (T) was also given out. As shown in Fig. 3c, the temperature was varied $0-95^{\circ} \mathrm{C}$, keeping other parameters constant for studying temperature effect of the substrate. With the increasing of the temperature, the reaction rate $(\mathrm{k})$ increased gradually. This illustrated that reaction rate was affected significantly by the temperature. The phenomenon mainly stems from the fact that random movement of molecules was obvious with the increase of temperature. When temperature rose to $95^{\circ} \mathrm{C}, \mathrm{Ag}$ NPs catalyst still showed better catalytic performance. It declared that Ag NPs had high-temperature-resistance, without larger reunion between metal particles. And then Ag NPs' activity would not be affected. When the experiment was carried out in the absence of the $\mathrm{Ag}$ NPs catalyst, it was observed that $\mathrm{NaBH}_{4}$ showed almost no change over $16 \mathrm{~min}$. Therefore, it could be confirmed that the catalyst was providing a surface for the adsorption of both species (4-NP and $\mathrm{NaBH}_{4}$ reducing agent molecules), which increased the rate of degradation reaction by increasing electron transfer between these reacting molecules [36].
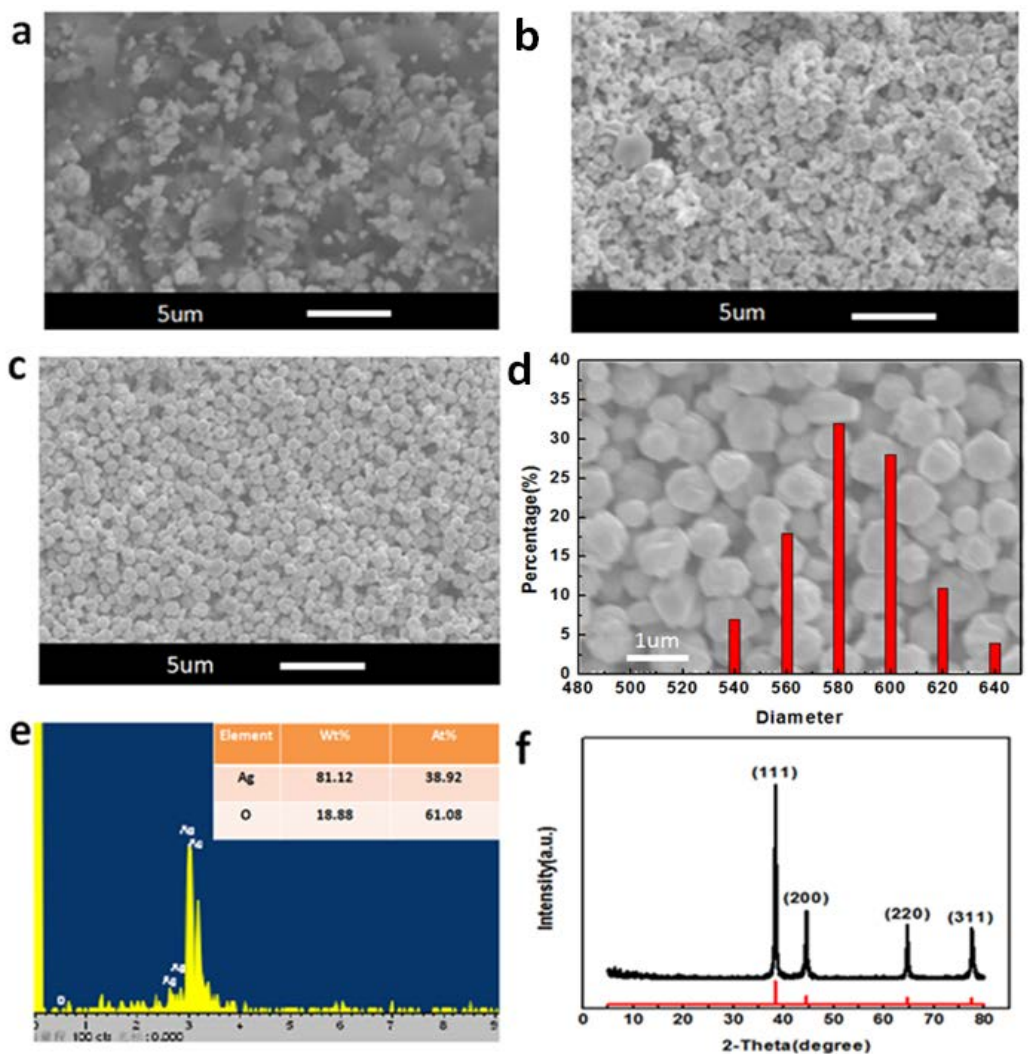

Figure 1. Low magnification SEM images of Ag NPs in microemulsion of different volume ratio (a) 10:1:2 (b) 10:10:2 (c) 10:5:2. (d) High magnification SEM images of Ag NPs in microemulsion with the volume ratio 10:5:2. Inset: particle size distribution of Ag NPs. (e) EDS spectrum of Ag NPs. Inset: element concentrations of Ag and O of Ag NPs. (f) Powder XRD patterns of Ag NPs. 

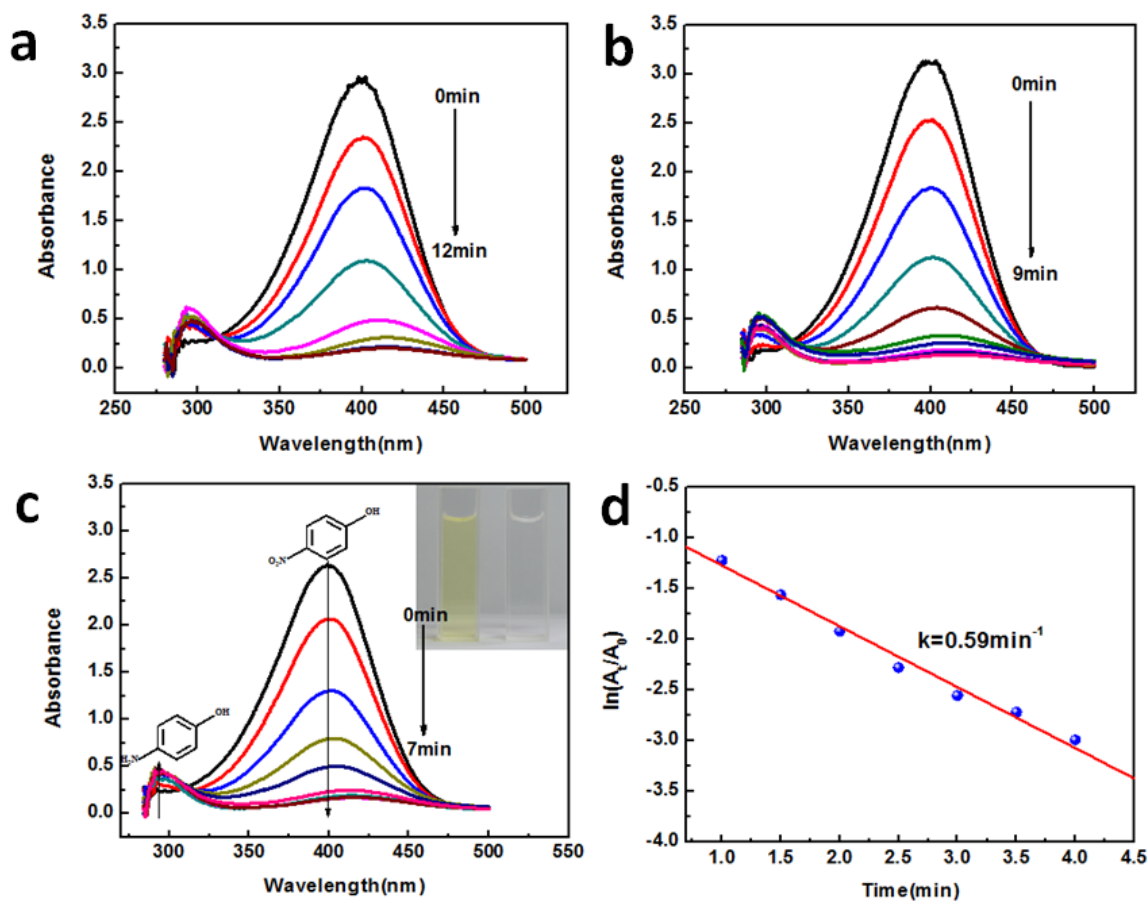

Figure 2. Time-dependent UV-vis spectra of the reaction solution in the presence of Ag NPs in microemulsion of different volume ratio (a) 10:1:2 (b) 10:10:2 (c) 10:5:2. Inset: Color change of 4-nitrophenol solution (left) before and (right) after the catalytic reaction. (d) Plot of $\ln (\mathrm{At} / \mathrm{A} 0)$ against the reaction time, corresponding to the reduction of 4-NP.

Table1. Recent studies on the reduction of 4-NP using various catalysts

\begin{tabular}{lll}
\hline Material & kapp $\left(\mathbf{m i n}^{-1}\right)$ & Ref. \\
\hline $\mathrm{Ag}-\mathrm{SiO}_{2} \mathrm{NWs}$ & 0.152 & {$[1]$} \\
$\mathrm{Ag} \mathrm{NPs} @ P G M A-S H$ & 0.236 & {$[30]$} \\
$\mathrm{CNC} @ P D A-\mathrm{Ag}$ & 0.2554 & {$[37]$} \\
$\mathrm{AgCit}$ & 0.0108 & {$[38]$} \\
acrylic acid -amidodiol/Ag hydrogel & 0.49 & {$[39]$} \\
$\mathrm{AgNP}-\mathrm{PG}-5 \mathrm{~K}$ & 0.33 & {$[40]$} \\
$\mathrm{Ag}-\mathrm{PMMA}$ & 0.049 & {$[41]$} \\
$\mathrm{Au} / \mathrm{AgNR} / \mathrm{SnO}_{2}$ & 0.50 & {$[42]$} \\
$\mathrm{Ag} \mathrm{NPs}$ & 0.59 & This work \\
\hline
\end{tabular}

Moreover, in reaction kinetics, the activation energy (Ea) can be obtained by the $\mathrm{k}$ under different temperature which is an empirical parameter describing the dependency of the $\mathrm{k}$ constant with temperature. A larger Ea value indicates higher sensitivity of the kinetic constant $\mathrm{k}$ to reaction temperature. The Ea values were calculated using the Arrhenius equation:

$$
\begin{aligned}
& \mathrm{k}_{\mathrm{app}}=\operatorname{Aexp}\left(-\frac{\mathrm{Ea}}{\mathrm{RT}}\right) \\
& \ln \mathrm{k}_{\mathrm{app}}=-\frac{\mathrm{Ea}}{\mathrm{RT}}+\ln \mathrm{A}
\end{aligned}
$$

where $\mathrm{A}$ is the Arrhenius factor, $\mathrm{k}$ is the rate constant at temperature $\mathrm{T}$ (in Kelvin), and $\mathrm{R}$ is the universal gas constant. From the slope of the plot of $\ln \mathrm{k}_{\text {app }}$ against 1/T, the Ea for the $\mathrm{Ag}$ NPs was determined to be $23.03 \mathrm{~kJ} \mathrm{~mol}^{-1}$, which was much smaller than some clad metal catalysts (the Ea for Ag based catalysts is 30-40 kJ mol ${ }^{-1}[37,44-46]$, the $\mathrm{Ea}$ for $\mathrm{Fe}_{3} \mathrm{O}_{4}$ based catalysts is 30-40 kJ mol-1 [47]). Usually, for 
surface-catalyzed reactions, the Ea value lies between $8.4 \mathrm{~kJ} \mathrm{~mol}^{-1}$ and $41.9 \mathrm{~kJ} \mathrm{~mol}^{-1}$ [48]. Thus, the results support the belief that the conversion of 4-NP to 4-AP occurred via surface catalysis. Moreover, the low Ea of Ag NPs was also in agreement with the superior photocatalytic activity of Ag NPs than other catalysts.
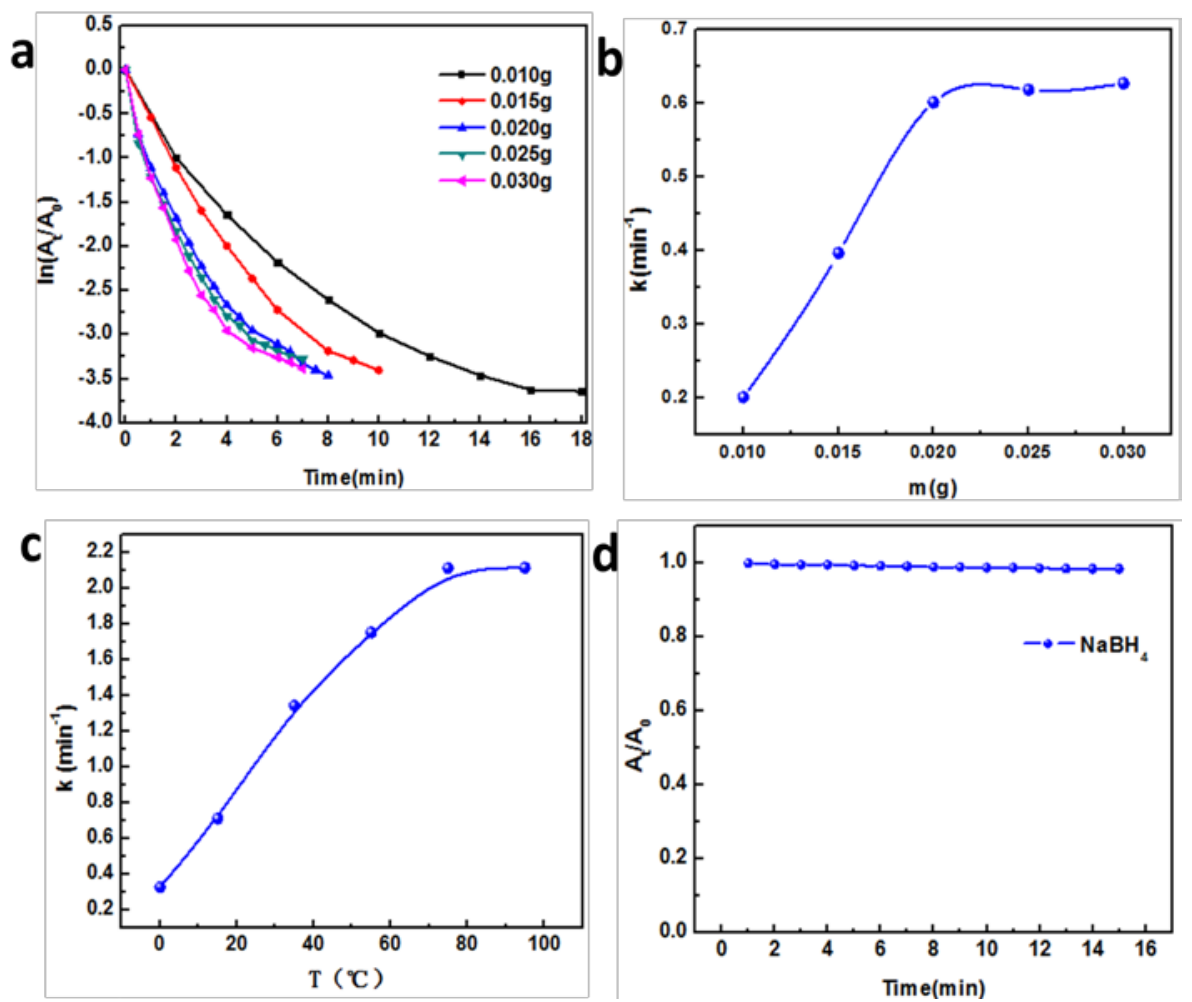

Figure 3. (a) Plot of $\ln \left(A_{t} / A_{0}\right)$ versus time $t$ for different silver quality in Ag NPs stock solutions. (b) Plot of $k$ versus the mass of catalyst Ag NPs. (c) Plot of k versus the reaction temperature. (d) Plot of $A_{t} / A_{0}$ vs reaction time for a mixture of 4-NP and $\mathrm{NaBH}_{4}$ without the addition of catalyst.
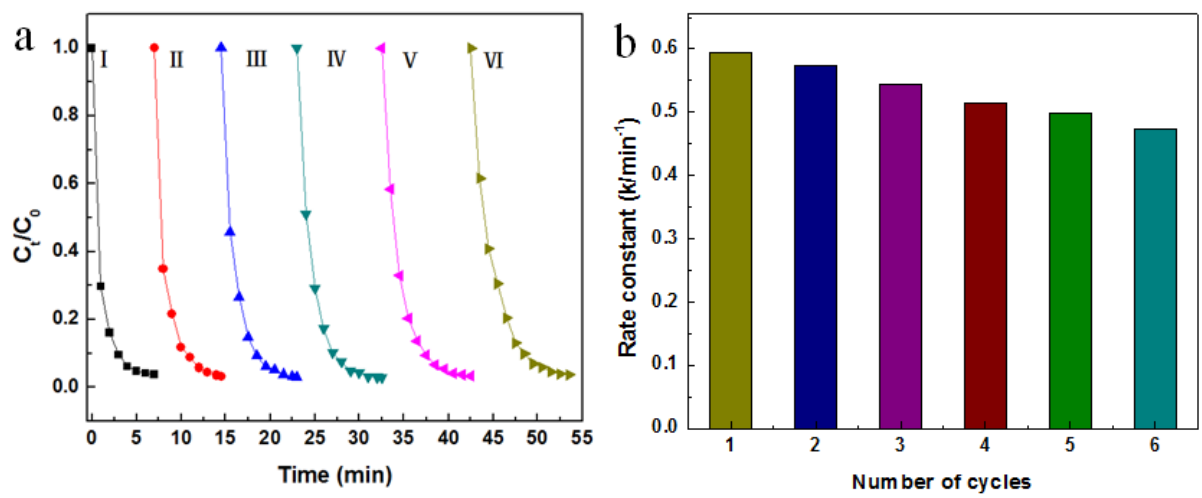

Figure 4. (a) Plot of $\mathrm{C}_{\mathrm{t}} / \mathrm{C}_{0}$ versus time (t) for the recycling of the catalyst. (b) Reusability of $\mathrm{Ag}$ NPs catalyst for the reduction of 4-NP with $\mathrm{NaBH}_{4}$.

To check the stability of Ag NPs catalyst, a recyclability test was carried out over six cycles. The catalyst was separated from the reaction solution after each cycle, and then washed and dried for the next run under identical condition. As shown in Fig. 4a and b, high yields were attained in all six reaction runs, indicative of the recoverable catalytic activity of the Ag NPs. We were aware of the slight decrease in conversion, which implied the possibility that the catalyst may leak off after many cycles in use. From the experimental results, the rate constant $(\mathrm{k})$ values of six successive cycles are $0.59 \mathrm{~min}^{-1}, 0.57 \mathrm{~min}^{-1}, 0.54$ 
$\mathrm{min}^{-1}, 0.51 \mathrm{~min}^{-1}, 0.50 \mathrm{~min}^{-1}, 0.47 \mathrm{~min}^{-1}$, respectively. Therefore, the as-prepared Ag NPs was proven to have excellent catalytic activity, reusability, and longterm stability, and thus to be a promising catalyst for the borohydride reduction reaction of $4-\mathrm{NP}$.

\subsection{Mechanism of Catalytic Activity Enhancement.}

The catalytic properties of Ag NPs were evaluated by reduction of 4-nitrophenol (4-NP) to 4-aminophenol (4-AP) with the present of $\mathrm{NaBH}_{4}$ (Fig. 5a). Fig. 5b shows the catalytic mechanisms for 4-NP reduction by $\mathrm{Ag}$ NPs in the presence of NaBH4. When Ag NPs were used for catalytic reduction, the electron transfer from $\mathrm{BH}_{4}^{-}$to $\mathrm{Ag}$ NPs and then to 4-NP was the first step. Herein, $\mathrm{BH}_{4}^{-}$was adsorbed onto the surface of $\mathrm{Ag}$ NPs to react and transfer electrons to Ag NPs [30]. And then the bare Ag NPs serve as catalyst to transfer electrons from $\mathrm{BH}_{4}^{-}$to nitrophenols, leading to the production of amino derivatives, 2-amion-4-nitrophenol and p-aminophenol [49].

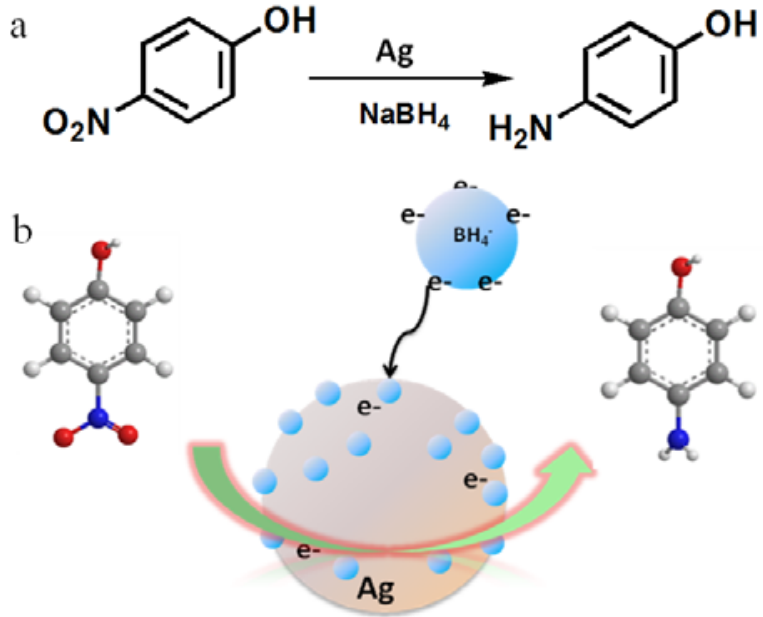

Figure 5. (a) schematic diagram for catalytic reaction of 4-NP. (b) Schematic illustration demonstrating the catalytic reaction mechanism of the reduction of 4-NP to 4-AP by aqueous solution of $\mathrm{NaBH}_{4}$ over $\mathrm{Ag} \mathrm{NPs}$ catalyst.

\section{Conclusions}

A simple, microemulsion technique for the in-situ synthesis of Ag NPs was proposed. A single metal of silver nanoparticles were prepared by a rather simple and fast method. The size and morphology of Ag NPs were highly controlled by microemulsion, which were obtained a granulum structure with a diameter of 540-640 nm. The Ag NPs exhibited high catalytic activity in the reduction of 4-NP in the presence of $\mathrm{NaBH}_{4}$, as a result of the high surface area and electronic properties of Ag NPs supports. Excellent reproducibility and stability were further confirmed. The silver nanoparticles can be extended to an important metalcatalyzed reduction in chemical industry, which is of great significance for the sustainable development.

\section{References}

1. Z. Hong, D. Tao, K. Z. Wen, and T. Y. Wei, "Natural Chrysotile-Based Nanowires Decorated with Monodispersed Ag Nanoparticles as a Highly Active and Reusable Hydrogenation Catalyst," J. Phys. Chem. C, vol. 119, no. 4, pp. $21465-21472,2015$.

2. A. Roy, B. Debnath, R. Sahoo, K. R. S. Chandrakumar, C. Ray, J. Jana, and T. Pal, "Enhanced Catalytic Activity of $\mathrm{Ag} / \mathrm{Rh}$ Bimetallic Nanomaterial: Evidence of an Ensemble Effect," J. Phys. Chem. C, vol.120, no. 10, pp. 5457-5467, 2016.

3. T. K. Sau, A. L. Rogach, F. Jackel, T. A. Klar, J. Feldmann, "Properties and Applications of Colloidal Nonspherical Noble Metal Nanoparticles," Adv. Mater. , vol. 22, no. 16, pp. 1805-1825, 2010. 
4. B. Baruah, G. J. Gabriel, M. J. Akbashev, and M. E. Booher, "Facile Synthesis of Silver Nanoparticles Stabilized by Cationic Polynorbornenes and Their Catalytic Activity in 4-Nitrophenol Reduction," Langmuir, vol. 29, no. 13, pp. 4225-4234, 2013.

5. S. Anandhakumar, M. Sasidharan, C. W. Tsao, and A. M. Raichur, "Tailor-Made Hollow Silver Nanoparticle Cages Assembled with Silver Nanoparticles: An Efficient Catalyst for Epoxidation," ACS Appl. Mater. Interfaces, vol. 6, no. 5, pp. 3275-3281, 2014.

6. M. O. Ansari, M. M. Khan, S. A. Ansari, K. Raju, J. Lee, and M. H. Cho, "Enhanced Thermal Stability under DC Electrical Conductivity Retention and Visible Light Activity of $\mathrm{Ag} / \mathrm{TiO}_{2} @$ Polyaniline Nanocomposite Film," ACS Appl. Mater. Interfaces, vol. 6, no. 11, pp. 8124-8133, 2014.

7. J. H. Yang, Y. Li, L. H. Zu, L. M. Tong, G. L. Liu, Y. Qin, and D. L. Shi, "Light-Concentrating Plasmonic Au Superstructures with Significantly Visible-Light-Enhanced Catalytic Performance, "ACS Appl. Mater. Interfaces, vol. 7, no. 15, pp. 8200-8208, 2015.

8. H. Song, R. M. Rioux, J. D. Hoefelmeyer, R. Komor, K. Niesz, M. Grass, P. D. Yang, G. A. Somorjai, "Hydrothermal Growth of Mesoporous SBA-15 Silica in the Presence of PVP-Stabilized Pt Nanoparticles: Synthesis, Characterization, and Catalytic Properties," J. Am. Chem. Soc. , vol. 128, no. 9, pp. 3027-3037, 2006.

9. G. J. Leong, A. Ebnonnasir, M. C. Schulze, M. B. Strand, C. Ngo, D. Maloney, S. L. Frisco, H. N. Dinh, B. Pivovar, G. H. Gilmer, "Shape-Directional Growth of Pt and Pd Nanoparticles," Nanoscale, vol. 6, no. 19, pp. 11364-11371, 2014.

10. S. Y. Li, D. L. Lin, J. F. Zhou, and L. S. Zha, "Preparation of Silver Nanoparticles Loaded Photoresponsive Composite Microgels and Their Light-Controllable Catalytic Activity," J. Phys. Chem. C, vol. 120, no. 9, pp. 4902-4908, 2016.

11. J. L. Zhang, M. X. Zhang, K. J. Tang, F. Verpoort, T. L. Sun, "Polymer-Based Stimuli-Responsive Recyclable Catalytic Systems for Organic Synthesis," Small, vol.10, no. 1, pp. 32-46, 2014.

12. M. V. Canamares, J. V. Garcia-Ramos, J. D. Gomez-Varga, C. Domingo, S. Sanchez-Cortes, "Comparative study of the morphology, aggregation, adherence to Glass, and surface-enhanced raman scattering activity of silver nanoparticles prepared by chemical reduction of $\mathrm{Ag}^{+}$using citrate and hydroxylamine," Langmuir, vol. 21, no. 18, pp. 8546-8553, 2005.

13. P. L. Redmond, A. J. Hallock, L. E. Brus, "Electrochemical ostwald ripening of colloidal Ag particles on conductive substrates," Nano Lett. , vol. 5, no. 1, pp. 131-135, 2005.

14. F. Liao, Z. F. Wang, and X. Q. Hu, "Shape-Controllable Synthesis of Dendritic Silver Nanostructures at Room Temperature," Colloid J. , vol. 73, no. 4, pp. 504-508, 2010.

15. F. Liao Z. F. Wang X. Q. Hu, "Growth of different morphologies of silver submicrostructures: the effect of concentrations and pH," Springer, vol. 17, no. 2, pp. 81-86, 2011.

16. F. Liao, Z. F. Wang and X. P. Sun, "A Novel Method Self-Assemble Silver Nanowires at Room Temperature," Nano-Metal Chemistry, vol. 42, no. 3, pp. 325-328, 2012.

17. Z. F. Wang, F. Liao, T. T. Guo, S.W. Yang, "Chunmei Zeng Synthesis of crystalline silver nanoplates and their application for detection of nitrite in foods," Journal of Electroanalytical Chemistry, vol. 664, no. 1, pp. 135-138, 2012 .

18. J. Bahadur, D. Sen, S. Mazumder, P. U. Sastry, B. Paul, H. Bhatt, S. G. Singh, "One-step fabrication of thermally stable $\mathrm{TiO}_{2} / \mathrm{SiO}_{2}$ nanocomposite microspheres by evaporation-induced self-assembly," Langmuir, vol. 28, no. 31, pp. 11343-11353, 2012.

19. J. Xu, J. P. Ge, Y. D. Li, "Solvthermal synthesis of monodisperse PbSe nanocrystals," J. Phys. Chem. B, vol. 110, no. 6, pp. 2497-2501, 2006.

20. C. Dumas and C. J. Meledandri, "Insights into the Partitioning Behavior of Secondary Surfactants in a Microemulsion-Based Synthesis of Metal Nanoparticles: A DLS and 2D NMR Spectroscopic Investigation," Langmuir, vol. 31, no. 26, pp. 7193-7203, 2015.

21. K. Tong, C. H. Zhao, Z. C. Sun, and D. J. Sun, "Formation of Concentrated Nanoemulsion by W/O Microemulsion Dilution Method: Biodiesel, Tween 80, and Water System," ACS Sustainable Chem. Eng., vol. 3, no. 12, pp. 3299-3306, 2015.

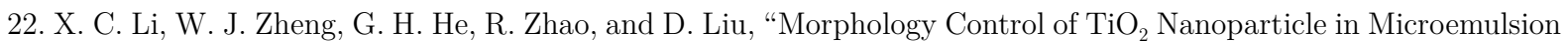
and Its Photocatalytic Property," ACS Sustainable Chem. Eng., vol. 2, no. 2, pp. 288-295, 2014.

23. C. Solans, P. Izquierdo, J. Nolla, N. Azemar, M. Garcia-Celma, "Nano-emulsion," J. Curr. Opin. Colloid Interface 
Sci. , vol. 10, no. 3-4, pp. 102-110, 2005.

24. M. H. Zhu, P. L. Chen, and M. H. Liu, "Ag/AgBr/Graphene Oxide Nanocomposite Synthesized via Oil/Water and Water/Oil Microemulsions: A Comparison of Sunlight Energized Plasmonic Photocatalytic Activity," Langmuir, vol. 28, no. 7, pp. 3385-3390, 2012.

25. A. K. Ganguli, A. Ganguly, S. Vaidya, "Mircroemulsion-based synthesis of nanocrystalline materials," Chem. Soc. Rev., vol. 39, no. 2, 474-485, 2010.

26. R. K. Gupta, M. Dubey, P. Z. Li, Q. Xu, and D. S. Pandey. "Size-Controlled Synthesis of Ag Nanoparticles Functionalized by Heteroleptic Dipyrrinato Complexes Having meso-Pyridyl Substituents and Their Catalytic Applications," Inorg. Chem., vol. 54, no. 6, pp. 2500-2511, 2015.

27. T. R. Lin, J. Wang, L.Q. Guo, and F. F. Fu. "Fe $\mathrm{O}_{4} @ \mathrm{MoS}_{2}$ Core-Shell Composites: Preparation, Characterization, and Catalytic Application," J. Phys. Chem. C, vol. 119, no. 24, 13658-13664, 2015.

28. Y. Ma, Y. H. Ni, F. Guo, and N. N. Xiang, "Flowerlike Copper(II)-Based Coordination Polymers Particles: Rapid Room-Temperature Fabrication, Influencing Factors, and Transformation toward $\mathrm{CuO}$ Microstructures with Good Catalytic Activity for the Reduction of 4-Nitrophenol," Cryst. Growth Des. , vol. 15, no. 5, pp. 2243-2252, 2015.

29. J. J. Zhou, C. X. Wang, P. Wang, P. B. Messersmith, and H. W. Duan, "Multifunctional Magnetic Nanochains: Exploiting Self-Polymerization and Versatile Reactivity of Mussel-Inspired Polydopamine," Chem. Mater., vol. 27, no. 8, pp. 3071-3076, 2015.

30. W. C. Zhang, Y. Sun, and L. Zhang, "In Situ Synthesis of Monodisperse Silver Nanoparticles on SulfhydrylFunctionalized Poly(glycidyl methacrylate) Microspheres for Catalytic Reduction of 4-Nitrophenol," Ind. Eng. Chem. Res., vol. 54, no. 25 6480-6488, 2015.

31. Z. H. Cao, H. N. Chen, S. D. Zhu, W. W. Zhang, X. F. Wu, G. R. Shan, U. Ziener, and D. M. Qi, "Preparation of Janus Pd/SiO $\mathrm{S}_{2}$ Nanocomposite Particles in Inverse Miniemulsions," Langmuir, vol. 31, no. 14, pp. 4341-4350, 2015.

32. X. Yu, G. Cheng, M. D. Zhou, and S. Y. Zheng, "On-Demand One-Step Synthesis of Monodisperse Functional Polymeric Microspheres with Droplet Microfluidics," Langmuir, vol. 31, no. 13, pp. 3982-3992, 2015.

33. W. Xiao, Y. H. Zhang, and B. T. Liu, "Raspberrylike $\mathrm{SiO}_{2} @$ Reduced Graphene Oxide@AgNP Composite Microspheres with High Aqueous Dispersity and Excellent Catalytic Activity," ACS Appl. Mater. Interfaces, vol. 7, no. 11, pp. 6041-6046, 2015.

34. J. H. Yang, Y. Li, L. H. Zu, L. M. Tong, G. L. Liu, Y. Qin, and D. L. Shi, "Light-Concentrating Plasmonic Au Superstructures with Significantly Visible-Light-Enhanced Catalytic Performance," ACS Appl. Mater. Interfaces, vol. 7, no. 15, pp. 8200-8208, 2015.

35. P. Zhang, R. Li, Y. M. Huang, and Q. W. Chen, "A Novel Approach for the in Situ Synthesis of Pt-Pd Nanoalloys Supported on $\mathrm{Fe}_{3} \mathrm{O}_{4} @ \mathrm{C}$ Core-Shell Nanoparticles with Enhanced Catalytic Activity for Reduction Reactions," ACS Appl. Mater. Interfaces, vol. 6, no. 4, pp. 2671-2678, 2014.

36. M. Kumar and S. Deka, "Multiply Twinned AgNi Alloy Nanoparticles as Highly Active Catalyst for Multiple Reduction and Degradation Reactions," ACS Appl. Mater. Interfaces, vol. 6, no. 18, pp. 16071-16081, 2014.

37. J. T. Tang, Z. Q. Shi, R. M. Berry, and K. C. Tam, "Mussel-Inspired Green Metallization of Silver Nanoparticles on Cellulose Nanocrystals and Their Enhanced Catalytic Reduction of 4-Nitrophenol in the Presence of ß-Cyclodextrin," Ind. Eng. Chem. Res. , vol. 54, no. 13, pp. 3299-3308, 2015.

38. J. Kumar, R. Mallampati, A. Adin, and S. Valiyaveettil, "Functionalized Carbon Spheres for Extraction of Nanoparticles and Catalyst Support in Water," ACS Sustainable Chem. Eng., vol. 2, no. 12, pp. 2675-2682, 2014.

39. R. K. Narayanan and S. J. Devaki, "Brawny Silver-Hydrogel Based Nanocatalyst for Reduction of Nitrophenols: Studies on Kinetics and Mechanism," Ind. Eng. Chem. Res., vol. 54, no. 4, pp. 1197-1203, 2015.

40. B. Baruah, G. J. Gabriel, M. J. Akbashev, and M. E. Booher, "Facile Synthesis of Silver Nanoparticles Stabilized by Cationic Polynorbornenes and Their Catalytic Activity in 4-Nitrophenol Reduction," Langmuir, vol. 29, no. 13, pp. 4225-4234, 2013.

41. M. D. Prasad and M. G. Krishna, "Facile Green Chemistry-Based Synthesis and Properties of Free-Standing Au-and Ag-PMMA Films," ACS Sustainable Chem. Eng., vol. 2, no. 6, pp. 1453-1460, 2014.

42. N. Zhou, L. Polavarapu, Q. Wang, and Q. H. Xu, "Mesoporous $\mathrm{SnO}_{2}$-Coated Metal Nanoparticles with Enhanced Catalytic Efficiency," ACS Appl. Mater. Interfaces, vol. 7, no. 8, pp. 4844-4850, 2015.

43. Y. Li, J. Y. Lan, J. J. Liu, J. F. Yu, Z. P. Luo, W. X. Wang, and L. Y. Sun, "Synthesis of Gold Nanoparticles on Rice Husk Silica for Catalysis Applications," Ind. Eng. Chem. Res. , vol. 54, no. 21, pp. 5656-5663, 2015.

44. J. R. Chiou, B. H. Lai, K. C. Hsu, D. H. Chen, "One-pot green synthesis of silver/iron oxide composite 
nanoparticles for 4-nitrophenol reduction," J. Hazard. Mater., vol. 394, no. 1, pp. 248-249, 2013.

45. S. Wunder, F. Polzer, Y. Lu, Y. Mei, M. Ballauff, "Kinetic Analysis of the Catalytic Reduction of 4-Nitrophenol by Metallic Nanoparticles," J. Phys. Chem. C, vol. 118, no. 32, pp. 18618-18625, 2014.

46. L. Q. Xu, B. S. M. Yap, R. Wang, K.-G. Neoh, E. T. Kang, G. D. Fu, "Catecholamine-Induced Electroless Metallization of Silver on Silica@Polymer Hybrid Nanospheres and Their Catalytic Applications," Ind. Eng. Chem. Res., vol. 53, no. 8, pp. 3116-3124, 2014.

47. M. Zhu, C. Wang, D. Meng, G. Diao, "In situ synthesis of silver nanostructures on magnetic $\mathrm{Fe}_{3} \mathrm{O}_{4} @ \mathrm{C}$ core-shell nanocomposites and their application in catalytic reduction reactions," J. Mater. Chem. A, vol. 1, no. 6, pp. 2118-2125, 2013

48. S. Saha, A. Pal, S. Kundu, S. Basu, T. Pal, "Photochemical Green Synthesis of Calcium-Alginate-Stabilized Ag and Au Nanoparticles and Their Catalytic Application to 4-Nitrophenol Reduction," Langmuir, vol. 26, no. 4, pp. 2885-2893, 2010.

49. F. H. Lin and R. A. Doong, "Bifunctional $\mathrm{AuFe}_{3} \mathrm{O}_{4}$ Heterostructures for Magnetically Recyclable Catalysis of Nitrophenol Reduction," J. Phys. Chem. C, vol. 115, no. 14, pp. 6591-6598, 2011. 